\title{
ESTIMACIÓN MICROSCÓPICA DE EDAD A PARTIR DE LA ZONA CORTICAL DEL FÉMUR EN INDIVIDUOS ADULTOS: REVISIÓN METODOLÓGICA
}

\author{
Bárbara Desántolo* y Ana M. Inda \\ Cátedra de Citología, Histología y Embriología A. Facultad de Ciencias Médicas. Universidad Nacional de La Plata. La Plata. Argentina
}

PALABRAS CLAVE antropología forense; análisis histomorfométrico

\begin{abstract}
RESUMEN El principal aporte de la histología cuantitativa a la antropología ha sido la estimación de edad a la muerte en restos óseos humanos no documentados. Los procesos secuenciales de remodelación ósea permiten observar la asociación entre el número de osteonas y la edad cronológica, lo cual constituye la base primaria de los métodos histológicos de predicción de edad. El primer estudio sobre cambios en la microestructura ósea y su aplicación para el cálculo de la edad en esqueletos adultos fue desarrollado en
\end{abstract}

1965. Posteriormente, el mismo fue testeado en muestras independientes y modificado por varios investigadores que trataron de subsanar y ajustar algunos inconvenientes, sobre todo aquellos vinculados con la precisión y exactitud. El siguiente artículo de revisión tiene como objetivo discutir los principales métodos histológicos de estimación de edad aplicados a restos óseos humanos y sintetizar el estado actual del conocimiento al respecto. Rev Arg Antrop Biol 18(2), 2016. doi:10.17139/raab.2016.0018.02.04

KEYWORDS forensic anthropology, histomorphometric analysis

\begin{abstract}
The main contribution of quantitative histology to anthropology has been the estimation of age at death in undocumented human skeletal remains. Sequential bone remodeling processes allow us to observe the association between the number of osteons and chronological age, which constitutes the primary basis for histological age predicting methods. The first study on changes in bone microstructure and its application to age calculation in adult skeletons was
\end{abstract}

developed in 1965. Subsequently, it was tested in independent samples and modified by several researchers with the intention of rectifying and adjusting some drawbacks, especially those related to precision and accuracy. The following review article aims to discuss major histological age estimation methods applied to human remains and summarize the current state of knowledge in this area. Rev Arg Antrop Biol 18(2), 2016. doi:10.17139/raab.2016.0018.02.04
La mineralización de los tejidos duros -huesos $\mathrm{y}$ dientes- durante la vida de un individuo permite la preservación de partes del esqueleto después de la muerte del organismo, convirtiéndose en una de las mayores fuentes de información para la bioantropología, la osteología forense así como para la paleodemografía, la biología ósea, entre otras disciplinas. Lejos de su concepción tradicional como un elemento estático, el hueso es un tejido extremadamente dinámico y complejo que crece durante toda la vida de un organismo, cambia de tamaño, forma y posición (Enlow, 1982) en respuesta a una multiplicidad de estímulos ambientales, genéticos, nutricionales, metabólicos, hormonales y mecánicos (Kemkes-Grottenthaler, 2002). De esta manera se convierte en un documento persistente de los cambios que se producen durante la vida del individuo (White et al., 2012).

Así, una de las principales problemáticas a dilucidar en la reconstrucción bioantropológica de individuos o grupos humanos no documentados, es la estimación de edad a la muerte a partir de restos óseos (Lynnerup et al., 2006).
Estimación que se fundamenta en las sucesivas transformaciones experimentadas por el sistema esqueletal en su evolución ontogénetica, i.e durante su crecimiento y desarrollo (Scheuer, 2002). Por lo tanto, la estimación de la edad en restos no documentados implica la observación de una multiplicidad de rasgos morfológicos que se expresan durante los sucesivos estados de formación, maduración y consolidación del tejido óseo y dental, comparando la información obtenida con los cambios registrados en poblaciones recientes de edad conocida (Ferembach et al., 1980, Ubelaker, 1986, 2000). En otras palabras, la estimación de la edad biológi-

\footnotetext{
Financiamiento: Universidad Nacional de La Plata (M157).

*Correspondencia a: Bárbara Desántolo. Cátedra de Citología, Histología y Embriología A. Facultad de Ciencias Médicas. UNLP. 60 y $120 \mathrm{~s} / \mathrm{N}^{\circ} .1900$ La Plata. Buenos Aires. Argentina. E-mail: barbaradesantolo@fcnym.unlp.edu.ar
}

Recibido 6 Marzo 2015; aceptado 27 Julio 2015

doi:10.17139/raab.2016.0018.02.04 
ca se infiere a partir de variables correlacionadas con la edad cronológica.

Sin embargo, la relación entre edad biológica y cronológica no es constante ni lineal dado que los cambios biológicos no son uniformes y están influenciados, además, por diferentes factores intrínsecos (e.g. genes, sexo, ancestría) y extrínsecos (e.g. ambiente, estrés, alimentación, patologías y actividad física) provocando una gran variación dentro y entre poblaciones. Por lo tanto, la variabilidad en las poblaciones humanas, conlleva un cierto margen de error en la estimación de la edad biológica y se expresa en los distintos grados de precisión y exactitud (Schmitt et al., 2002; Krenzer, 2006). Se entiende por "precisión" al conjunto de medidas de una misma magnitud realizadas condiciones semejantes, es la medida de dispersión de los datos (Santo y Lecumberry, 2005) y por "exactitud", a la distancia entre el valor medido y el valor real (Ferrante y Cameriere, 2009), está relacionada con el sesgo de una estimación. Cuanto menor es el sesgo más exacta es una estimación (Christensen y Crowder, 2009).

Según White y colaboradores (2011) el grado de precisión y exactitud en la estimación, dependerá de los métodos aplicados según las categorías de edad y de los elementos esqueletales disponibles para el análisis. En el caso de subadultos, desde el nacimiento hasta la adolescencia, la edad se puede diagnosticar a través de la observación de la forma y el estado de metamorfosis de los centros de osificación (Fazekas y Kosa, 1978; Kosa, 1989; Schmeling et al., 2007), la formación y erupción dental (Hillson, 2005), la progresión en el cierre epifisial, como también, la longitud de los huesos largos (Scheuer y Black, 2000; Shaefer et al., 2009), fusión de la sincondrosis esfeno-occipital/basilar (Madeline y Elster, 1995) y la fusión clavícula-esternón (Schulz et al., 2005). Dichas variables se ven mucho menos afectadas por factores socioambientales respecto de las consideradas para los adultos. Es decir, que la pauta genética que la afecta es comparativamente mucho mayor (Luna, 2008).

En adultos, la estimación de la edad se realiza a través de métodos macroscópicos y microscópicos. Las técnicas macroscópicas -morfoscópicas y morfométricas- consisten en la observación directa de indicadores sobre el material óseo y dental. Son más rápidas y no implican destrucción del ma- terial pero su principal desventaja es que requieren de elementos esqueletales completos en la mayoría de los casos. Los principales métodos, universalmente aplicados, son: los cambios degenerativos de la sínfisis púbica (Meindl y Lovejoy, 1989; Brooks y Suchey, 1990), los de la superficie auricular del ilion (Lovejoy et al., 1985; Buckberry y Chamberlain, 2002; Osborne et al., 2004), cambios en la extremidad esternal de la IV costilla (Iscan et al., 1984; 1985; Yoder et al., 2001) y el grado de sinostosis de las suturas craneales (Meindl y Lovejoy, 1985). En el caso del desgaste dental (Lovejoy, 1985) y los cambios degenerativos, que afectan a la estructura ósea (columna vertebral, articulaciones, entre otros), la naturaleza de su variación (dieta, prácticas culturales, sexo, factores genéticos) ha dificultado su medición y correlación con la edad del individuo, por lo tanto las estimaciones, a partir de ambos indicadores, resultan poco confiables.

En cuanto a los métodos microscópicos, la histomorfometría o histología cuantitativa (Robling y Stout, 2008), ofrece estimaciones más precisas y efectivas para adultos mayores, en contraste con la mayoría de los métodos basados en la morfología esqueletal (Franklin, 2010). Además, son de gran utilidad aun cuando los restos óseos (arqueológicos o modernos) se encuentren fragmentados o incompletos, ya que por su estado de conservación resulta difícil estimar la edad macroscópicamente (Bednarek, 2008; Franklin, 2010; Streeter, 2012).

Sin embargo, estos métodos presentan ciertas desventajas con respecto a los macroscópicos: requieren de más tiempo, equipamiento, conocimiento específico de técnicas histológicas e implican destrucción parcial del material (Ubelaker, 2008). Además, otro factor que puede afectar el análisis microscópico, es el grado de preservación del tejido óseo debido a la acción de agentes tafonómicos y/o diagenéticos (ver Bell, 2012).

Por consiguiente, la interpretación de la información codificada en la microestructura ósea requiere un conocimiento avanzado de la biología que subyace a la creación de estructuras histomorfológicas (Stout y Crowder, 2012). Esta información es crucial para la estimación histológica de la edad que constituye el aporte más común de la histología a la investigación antropológica y forense.

El siguiente artículo de revisión tiene como objetivo discutir los principales métodos histo- 
lógicos de estimación de edad, aplicado a restos óseos humanos y sintetizar el estado actual del conocimiento al respecto.

\section{Estimación histomorfométrica de la edad}

La histomorfometría es la cuantificación de la microestructura del tejido óseo, donde las características histomorfológicas se describen en términos de números por unidad o tamaño de área. Así, a través del conteo y la medición de las características histomorfológicas puede obtenerse información acerca del recambio óseo (remodelación), de la microarquitectura y de la actividad celular dinámica y estática (Stout y Crowder, 2012).

En el campo antropológico y forense, el análisis histomorfométrico ha sido y es comúnmente aplicado en la estimación de la edad a la muerte (Kerley, 1965; Singh y Gunberg, 1970; Stout, 1988; Ericksen, 1991; Yoshino et al., 1994; Watanabe et al., 1998; Vasallo, 2000; Cho et al., 2002; Maat et al., 2006; Nor et al., 2006; Han et al., 2009; Gomes et al., 2014), en estudios tafonómicos y diagenéticos (Bell, 2012), diferenciación taxonómica (Hillier y Bell, 2007; Martiniaková et al., 2006; Mulhern y Ubelaker, 2012; Crescimanno y Stout, 2012), análisis de actividad ocupacional y biomecánica (Robling y Stout, 2008; Skedros, 2012) entre otros.

La estimación histológica de la edad se basa en el análisis del grado de remodelación ósea. La reabsorción y formación de hueso es llevada a cabo por la acción sucesiva de osteoclastos y osteoblastos en zonas delimitadas denominadas Unidades Multicelulares Básicas $\left(\mathrm{BMU}_{\mathrm{S}}\right)$ (Frost, 1985) o Unidades de Remodelación Ósea (BRU) (Parfitt, 1979), el tejido óseo resultante de la acción de cada BMU se denomina Unidad Estructural Ósea (BSU) (Robling y Stout, 2008). En el hueso cortical estas unidades microscópicas discretas, definibles y cuantificables están representadas por los sistemas de Havers u osteonas secundarias y, en el hueso trabecular por osteonas trabeculares o paquetes óseos (Bruce et al., 1998) (para una mayor comprensión de las características histofisiológicas e histomorfológicas véase Robling y Stout, 2008; Stout y Crowder, 2012).

Dado que la remodelación es un proceso que transcurre durante toda la vida, desde la etapa fetal hasta la muerte (Martin et al., 1998), permite ob- servar la asociación entre el número de osteonas y la edad cronológica constituyendo la base primaria de los métodos histológicos de estimación de edad (Stout, 1989). De esta manera y con el incremento de la edad, los componentes originales del hueso cortical (e.g. hueso laminar circunferencial, osteonas primarias) serán gradualmente reemplazados por nuevas estructuras (e.g. osteonas secundarias o sistemas de Havers). Posteriormente, en una edad avanzada, el proceso de remodelado continúa no sólo a expensas del hueso laminar y de las osteonas primarias, sino también de las osteonas secundarias preexistentes, formando así fragmentos osteonales (Ubelaker, 2005).

En los individuos mayores, la cantidad relativa de osteonas eliminadas aumenta hasta llegar a una asíntota. La asíntota de la Densidad Poblacional Osteonal (OPD) (número de osteonas completas y fragmentarias por $\mathrm{mm}^{2}$ ) varía en los diferentes huesos dependiendo de ciertos factores como la tasa de activación de la remodelación, el diámetro cortical y el tamaño osteonal (Cho et al., 2002; Stout y Crowder, 2012). En el caso del fémur, la asíntota de la OPD es alrededor de 50/ $\mathrm{mm}^{2}$ (Kerley, 1965; Frost, 1987) y se produce a una edad relativamente mayor, a partir de los 50 años, debido al considerable tamaño de sus áreas corticales. Constituyendo, de esta manera, una pieza clave para la estimación etaria en individuos de edad avanzada (Robling y Stout, 2008).

Asimismo, se ha demostrado que la mitad anterior de la diáfisis femoral es el área más resistente al deterioro tafonómico después de un periodo prolongado de entierro, es fácilmente identificable y además, la superficie cortical al no tener inserciones musculares presenta cierto grado de estabilidad biomecánica (Kerley, 1965; Ericksen, 1991; Maat et al., 2006, Keough et al., 2009, Nor et al., 2014).

De esta manera, los componentes microestructurales producto de la remodelación intracortical, osteonas secundarias y sus derivados (osteonas tipo I, tipo II, fragmentos osteonales, entre otros), constituyen las variables que mejor predicen la edad y por lo tanto, son esgrimidas en los métodos histológicos de estimación de la edad (Fig. 1) (Stout y Crowder, 2012). La mayoría de los métodos propuestos, con resultados promisorios, han sido desarrollados principalmente a partir de huesos largos tal como el fémur como se detalla a continuación (Tabla1). 


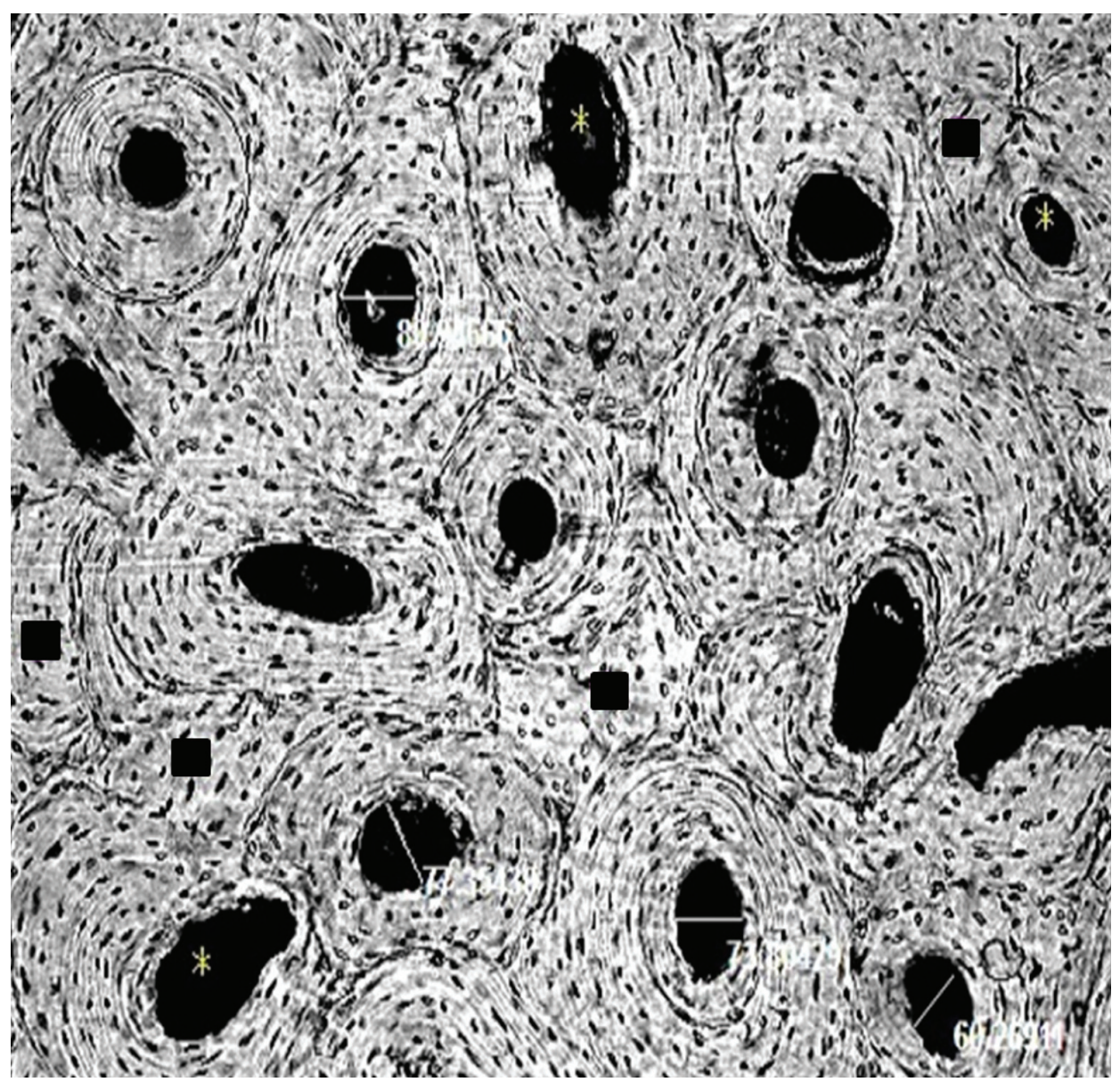

Fig. 1. Microfotografía - Tejido cortical de fémur (100x). Individuo femenino, 63 años. Referencias: círculos: osteonas secundarias completas (N. On.), estrella color negro: osteonas fragmentarias (N. On. Fg.), línea blanca: diámetro conducto de Havers (Hav. Can).

TABLA 1. Métodos de estimación de edad a partir de fémur

\begin{tabular}{|c|c|c|c|}
\hline Métodos & $\begin{array}{c}\text { Muestra (N), Rangos de edad } \\
\text { (x), Sexo }\left(0^{\dagger}: \bigcirc: ?\right)\end{array}$ & Variables Relevadas & S.E.E \\
\hline $\begin{array}{l}\text { Kerley, 1965, Kerley y } \\
\text { Ubelaker, } 1978\end{array}$ & $\begin{array}{l}\text { (67) 0-95 (41.6) 43:17:7 } \\
\text { Norteamericanos } \\
\text { descendientes de europeos } \\
\text { y africanos }\end{array}$ & $\begin{array}{l}\text { 1) Osteonas fragmentarias } \\
\text { 2) Osteonas completas } \\
\text { 3) Porcentaje de hueso laminar } \\
\text { circunferencial }\end{array}$ & $\begin{array}{l}9.39-13.85 \\
6.98-12.52\end{array}$ \\
\hline
\end{tabular}

\begin{tabular}{llll}
\hline Ahlqvist y Damsten, 1969 & $\begin{array}{l}\text { (20) (55.4) } \\
\text { Individuos provenientes de } \\
\text { autopsias sin especificación } \\
\text { étnica }\end{array}$ & 1) Porcentaje de hueso remodelado & $6.71-6.79$ \\
\hline Singh y Gunberg, 1970 & $\begin{array}{l}\text { (33) 39-87 (62.3) 33:0:0 } \\
\text { Individuos provenientes de } \\
\text { autopsias sin especificación } \\
\text { étnica }\end{array}$ & $\begin{array}{l}\text { 1) Número de osteonas } \\
\text { osteona 3) Promedio del diámetro } \\
\text { de los conductos de Havers }\end{array}$ & $3.24-5.01$ \\
\hline
\end{tabular}


TABLA 1. Métodos de estimación de edad a partir de fémur

\begin{tabular}{|c|c|c|c|}
\hline Métodos & $\begin{array}{c}\text { Muestra (N), Rangos de edad } \\
\text { (x), Sexo }(\delta: q: ?)\end{array}$ & Variables Relevadas & S.E.E \\
\hline Thompson, 1979 & $\begin{array}{l}\text { (116) 30-97 (71.7) 64:52:0 } \\
\text { Individuos de Nueva } \\
\text { Inglaterra }\end{array}$ & $\begin{array}{l}19 \text { variables histológicas y no } \\
\text { histológicas }\end{array}$ & $7.07-8.65$ \\
\hline Eriksen, 1991 & $\begin{array}{l}\text { (328) 14-97 (62.8) 174:154:0 } \\
\text { Individuos de origen } \\
\text { heterogéneo EE.UU, Chile y } \\
\text { República Dominicana }\end{array}$ & $\begin{array}{l}\text { 1) Osteonas secundarias } \\
\text { 2) Osteonas tipo II } \\
\text { 3) Osteonas fragmetnarias } \\
\text { 4) Espacios de resorción } \\
\text { 5) Canales no haversianos } \\
\text { 6) Porcentaje promedio de hueso } \\
\text { circunferencial } \\
\text { 7) Porcentaje promedio de hueso } \\
\text { osteonal } \\
\text { 8) Porcentaje promedio de osteonas } \\
\text { fragmentarias }\end{array}$ & $10.08-12.21$ \\
\hline Watanabe, 1998 & $\begin{array}{l}\text { (98) 2-93 72:26:0 } \\
\text { Individuos japoneses }\end{array}$ & $\begin{array}{l}\text { 1) área osteonal } \\
\text { 2) perímetro osteonal } \\
\text { 3) diámetro máximo y mínimo de } \\
\text { osteonas y canales haversianos } \\
\text { 4) número de osteonas tipo II } \\
\text { 5) número de osteonas } \\
\text { fragmentarias }\end{array}$ & $4.88-6.39$ \\
\hline Nor et al., 2006 & $\begin{array}{l}(36) ?(53) 50: 14: 0 \\
\text { Individuos de Malasia }\end{array}$ & $\begin{array}{l}\text { 1) Espesor cortical } \\
\text { 2) osteonas completas } \\
\text { 3) área de los conductos de Havers } \\
\text { 4) diámetro de los canales de } \\
\text { Havers entre otras. }\end{array}$ & 14.04 \\
\hline Maat et al., 2006 & $\begin{array}{l}\text { (162) 15-96 ? 86:76:0 } \\
\text { Muestra contemporánea de } \\
\text { Holanda }\end{array}$ & $\begin{array}{l}\text { 1) Cantidad de hueso laminar no } \\
\text { remodelado }\end{array}$ & 9.16-10.06 \\
\hline Han et al., 2009 & $\begin{array}{l}\text { (72) 35-94 (68.5) 44:28:0 } \\
\text { Muestra contemporánea de } \\
\text { Korea }\end{array}$ & $\begin{array}{l}\text { 1) Densidad poblacional osteonal } \\
\text { 2) Tamaño promedio osteonal } \\
\text { 3) diámetro promedio de los } \\
\text { canales de Havers } \\
\text { 4) ancho cortical }\end{array}$ & $6.65-6.99$ \\
\hline Keough et al., 2009 & $\begin{array}{l}\text { (146) 19-82 (51.7) 105:41:0 } \\
\text { Muestra contemporánea de } \\
\text { Sudafrica }\end{array}$ & $\begin{array}{l}\text { 1) Número total de osteonas } \\
\text { 2) Porcentaje de hueso no } \\
\text { remodelado } \\
\text { 3) Número total de canales no } \\
\text { haversianos } \\
\text { 4) Porcentaje promedio de hueso } \\
\text { fragmentado }\end{array}$ & $13.31-14.04$ \\
\hline Vasallo et al., 2000 & $\begin{array}{l}\text { (20) } 15-98 \\
\text { Muestra contemporánea de } \\
\text { Argentina }\end{array}$ & $\begin{array}{l}\text { 1) Porcentaje de osteonas } \\
\text { fragmentarias }\end{array}$ & $\mathrm{N} / \mathrm{E}$ \\
\hline Desántolo, 2013 & $\begin{array}{l}\text { (71) 22-91 (69.86) 89:54:0 } \\
\text { Colección osteológica } \\
\text { Rómulo Lambre - Argentina }\end{array}$ & $\begin{array}{l}\text { 1) Número de osteonas } \\
\text { fragmentarias } \\
\text { 2) Densidad poblacional osteonal } \\
\text { 3) Porcentaje de osteonas } \\
\text { fragmentarias } \\
\text { 4) Osteonas completas } \\
\text { 5) Diámetro promedio de los } \\
\text { conductos de Havers }\end{array}$ & $1.43-9.93$ \\
\hline
\end{tabular}

Muestra (N), Rangos de edad (x), Sexo ( $\bigcirc$ : masculino,, : femenino, ?: indeterminado), S.E.E: Error estándar de la estimación 


\section{Métodos histológicos en huesos largos, un repaso histórico}

El primer estudio sobre cambios en la microestructura ósea y su aplicación al cálculo de la edad en esqueletos adultos fue propuesto por Kerley (1965) quien desarrolló un método basado en el análisis microscópico del tejido cortical de los huesos largos. Para ello realizó 126 cortes transversales en la mitad de la diáfisis del fémur, tibia y peroné. La muestra estuvo integrada por individuos norteameamericanos descendientes de europeos y africanos, de ambos sexos (88 masculinos y 29 femeninos) de edades entre 0 y 95 años. En cada una de las secciones desgastadas delimitó y examinó cuatro campos circulares por debajo del periostio: anterior, posterior, medial y lateral, definiendo y cuantificando cuatro variables microestructurales. 1- Osteonas completas o sistema de Havers: canal vascular rodeado concéntricamente por hueso laminar. En la periferia de la osteona se encuentra la línea reversa que marca el área de actividad osteoclástica y de formación de nuevo hueso.2- Osteonas fragmentarias: fragmentos de antiguas osteonas producto de la remodelación, sin conducto de Havers. 3- Porcentaje de hueso laminar circunferencial: se compone de bandas o laminillas paralelas que se localizan en la parte exterior de la corteza. 4Sistemas no haversianos u osteonas primarias: incluye a todos los canales vasculares primarios o sistemas pseudo-haversianos. Se diferencian de las osteonas secundarias por la ausencia de laminillas. La observación microscópica fue realizada con un aumento 100x y el diámetro del campo fue de $1.25 \mathrm{~mm}$ (Kerley, 1965).

Los resultados de Kerley demostraron, en el fémur, un incremento lineal progresivo del número de osteonas secundarias $(\mathrm{r}=0.92)$ y fragmentarias $(\mathrm{r}=0.86)$ entre el nacimiento y los 95 años de edad. Por el contrario, el porcentaje de hueso laminar circunferencial y el número de canales no haversianos se mantiene alto durante la infancia, disminuye en la adolescencia y desaparece alrededor de los 55 años. Estos resultados se repiten en tibia y peroné. Años más tarde, Kerley y Ubelaker (1978) mejoraron las ecuaciones de regresión inicialmente propuestas, para ello reexaminaron las secciones de la muestra original, y advirtieron que las estimaciones calculadas a partir de osteonas fragmen- tarias eran más precisas en peroné e incrementaron el tamaño del campo a $1.62 \mathrm{~mm}$.

En 1969, Ahlqvist y Damsten trabajando con individuos provenientes de autopsias, sin especificación étnica, notaron ciertas dificultades para diferenciar osteonas completas de fragmentarias y para estimar el porcentaje de hueso lamelar en un campo visual circunferencial. Debido a esto propusieron modificaciones del método original: ubicaron cuatro campos rectangulares en la mitad de los cuadrantes planteados por Kerley, evitando el reborde elevado del eje posterior de la diáfisis femoral conocido como línea áspera y sólo cuantificaron el "porcentaje total de hueso remodelado" (osteonas completas y fragmentarias). Para la observación microscópica utilizaron un ocular reticulado de $10 \mathrm{~mm}^{2}$. El método fue testeado en una muestra de 20 secciones de fémur y el porcentaje obtenido en cada uno de los cuatro campos fue promediado para obtener un único resultado final. De todos modos, la proporción de osteonas secundarias y osteonas fragmentarias arrojó un error estándar (ES) en la estimación de \pm 6.71 años, comparado con el obtenido por Kerley para peroné de \pm 5.27 años. Pese a las recomendaciones, sus resultados fueron menos precisos que los obtenidos por Kerley.

Singh y Gunberg (1970) ofrecieron una nueva alternativa para estimar la edad a partir de la estructura histológica de fémur, tibia y mandíbula. La muestra analizada fue de 59 individuos provenientes de autopsias ( 52 masculinos y $7 \mathrm{fe}$ meninos) entre 39 y 87 años de edad. A diferencia de los estudios previos, examinaron secciones descalcificadas y sin descalcificar del borde posterior de la mandíbula y de la diáfisis de los huesos largos. En el caso del fémur, seleccionaron dos campos microscópicos circulares de 2 mm de diámetro, observados a 100X y elegidos al azar en la zona subperiosteal. Cuantificaron tres variables microestructurales: 1- número de osteonas secundarias, definidas sobre la presencia del conducto de Havers completo y de la línea de cemento. 2- número promedio de lamelas por osteonas, obtenido del total de lamelas de todas las osteonas observadas en dos campos microscópicos y 3- promedio del diámetro mínimo de los conductos de Havers, medido sólo en los conductos visualmente completos en el campo. Los resultados para huesos largos demuestran 
que el número total de osteonas (fémur $\mathrm{r}=0.94$ y tibia $\mathrm{r}=0.92$ ) y el diámetro del canal de Havers (fémur $\mathrm{r}=0.94$ y tibia $\mathrm{r}=0.93$ ) presentan mejores correlaciones que el número de laminillas por osteona (fémur $\mathrm{r}=0.89$ y tibia $\mathrm{r}=0.90$ ). En mandíbula, la correlación fue ligeramente mayor a la obtenida para fémur y tibia.

El modelo propuesto por Singh y Gunberg (1970), se basa exclusivamente en lo observado para la muestra masculina dada la escasa representación de individuos femeninos. De todos modos, al igual que Kerley (1965), no hallaron diferencias significativas entre sexos, probablemente debido a que ambas investigaciones son similares en el tamaño muestral de mujeres. En este caso, el error estándar de las estimaciones fue menor que el obtenido por Kerley y por Ahlqvist y Damsten (1969), pudiendo estimar la edad con una precisión de 2.58 años en el $67 \%$ y de 5,16 años en el 95\% de los individuos en el rango de edad entre 40 y 80 años (Keough 2007).En 1979, Thompson desarrolla un método que trata de superar los inconvenientes planteados en los estudios previos. Reduce al mínimo la destrucción de la pieza ósea, obteniendo para ello cilindros de hueso cortical (núcleos) de 0,4 cm de diámetro de la porción anterior de la diáfisis de fémur, tibia, húmero y cúbito. La muestra integrada por individuos de Nueva Inglaterra, incluyó 116 cadáveres (64 masculinos y 52 femeninos) de edad conocida, entre 30 y 97 años. Cuantificó 19 variables histológicas y no histológicas como el espesor cortical y la densidad ósea. Las secciones fueron examinadas con aumento 100x y los datos sometidos a análisis de regresión lineal a fin de seleccionar la variable, o combinación de variables, con menor error estándar y mayor coeficiente de determinación.

De este estudio surgieron varias ecuaciones de regresión demostrando que el área osteonal fue la variable más exacta en húmero $(\mathrm{ES}=6.2$ años). Además de demostrar que, después de los 50 años, comienza un proceso de constante degradación del espesor cortical y una disminución de la densidad mineral ósea diferencial entre ambos sexos (sexo femenino: $8 \%$ de espesor cortical y $10 \%$ de densidad ósea por década; sexo masculino: $4 \%$ de espesor cortical y $6 \%$ de densidad ósea por década) (Keough, 2007).

En 1991 Ericksen publica un nuevo método para estimar la edad a la muerte. Analiza seccio- nes delgadas, de $1.0 \mathrm{~cm}$ de ancho, de la porción anterior de la diáfisis femoral opuesta a la línea áspera. En cada sección selecciona cinco puntos equidistantes, que se fotografían, obteniéndose en cada uno de ellos un campo cuadrangular $\left(0.886 \mathrm{~mm}^{2}\right.$ y un área total de $\left.4.43 \mathrm{~mm}^{2}\right)$. La muestra estuvo integrada por 328 individuos (154 femeninos y 174 masculinos) entre 14 y 97 años, de origen heterogéneo (EE.UU., Chile y República Dominicana) con la finalidad de ampliar la composición étnica de la misma y por ende la aplicación de los resultados. Cuantifica ocho variables microestructurales: 1- osteonas secundarias $/ \mathrm{mm}^{2}$, 2- osteonas tipo $\mathrm{II} / \mathrm{mm}^{2}$, 3- osteonas fragmentarias $/ \mathrm{mm}^{2}, 4$ - espacios de resorción $/ \mathrm{mm}^{2}, 5$ - canales no haversianos $/ \mathrm{mm}^{2}$, 6- promedio del porcentaje de hueso circunferencial lamelar, 7- promedio del porcentaje de hueso osteonal y 8 - promedio del porcentaje de osteonas fragmentarias.

Además, realiza un análisis exploratorio de los factores responsables de las diferencias sexuales observadas durante el análisis estadístico. Propuso ecuaciones predictivas para individuos femeninos, masculinos y de sexos agrupados. La mayoría presentó un error estándar \pm 10 años, con un coeficiente de determinación $\left(\mathrm{r}^{2}\right)$ de 0.48 a 0.72 . En particular, las ecuaciones específicas para individuos masculinos $(E S=10.05)$ y femeninos $(E S=10.00)$ proveyeron mejores resultados que las ecuaciones para sexos agrupados $(\mathrm{ES}=10.08)$. Las diferencias entre sexos se observaron principalmente en dos variables microestructurales, osteonas completas y osteonas fragmentarias, lo cual, consecuentemente, demuestra la necesidad de generar fórmulas propias para cada sexo.

Años después, Watanabe y colaboradores (1998) establecieron fórmulas predictivas de estimación de edad basadas en una muestra japonesa. Analizaron 98 fémures, 72 masculinos (de 43 días a 92 años) y 26 femeninos (de 2 a 88 años). A diferencia de los estudios anteriores, las secciones fueron coloreadas con la técnica de Villanueva (Villanueva, 1974) y cada una de ellas fue registrada a través de microradiografías, cuantificándose las siguientes variables: área, perímetro, diámetro máximo y mínimo de osteonas y canales haversianos, número de osteonas tipo II y de osteonas fragmentarias. A su vez, los autores testearon una nueva varia- 
ble denominada "área del triángulo", delimitada por tres líneas adyacentes entre tres canales de Havers. Los parámetros obtenidos relacionados con las osteonas demostraron un alto coeficiente de correlación $(r>0.77)$ con la edad a diferencia de los hallados para los canales haversianos $(r>0.11)$. El error estándar de las estimaciones (3.16 a 11.50 años), según los autores, evidencia una mayor confiabilidad en el método respeto de los propuestos en otros trabajos.

Entrados en el siglo XXI y dada la evidencia de la variación poblacional en la dinámica de la remodelación ósea, lo cual puede dar lugar a estimaciones poco precisas cuando una ecuación predictiva para estimar la edad basada en una población es aplicada a las demás, se han propuesto nuevos métodos histológicos de estimación específicos para cada población.

Así, en Holanda, Maat y colaboradores (2006) desarrollaron ecuaciones predictivas basadas en una muestra contemporánea local. Examinaron segmentos de la diáfisis femoral de 162 individuos (86 masculinos y 76 femeninos) con edades comprendidas entre los 15 y 96 años. La observación microscópica fue realizada con un aumento 100X. Cuantificaron una sola variable (cantidad de hueso laminar no remodelado) $\mathrm{y}$ formularon ecuaciones predictivas. El coeficiente de determinación $\left(\mathrm{R}^{2}\right)$ de las ecuaciones osciló entre 0.60 y 0.79 y el error estándar (ES) de las estimaciones varió entre 9.16 y 10.6 años.

En Malasia, Nor y colaboradores (2006) elaboraron ecuaciones predictivas para húmero, cúbito, radio, fémur y tibia. La muestra seleccionada estuvo integrada por 64 individuos (50 masculinos y 14 femeninos) entre 21 y 78 años. Cuantificaron diez variables, cinco demostraron una correlación significativa con la edad. Los parámetros seleccionados fueron: conteo de osteonas $(\mathrm{r}=0.4)$, diámetro osteonal $(\mathrm{r}=0.39)$, área osteonal $(\mathrm{r}=-0.43)$, diámetro medular $(\mathrm{r}=0.25)$ y perímetro osteonal $(\mathrm{r}=-0.38)$. La ecuación propuesta para fémur arrojó un valor de $\mathrm{R}^{2}=0.59$ con un error estándar (ES) de 14.04 años.

En Sudáfrica, Keough y colaboradores (2009) desarrollaron estándares para estimar la edad, a nivel de la microestructura, en una población sudafricana. Para ello analizaron una muestra de 146 individuos de edad y sexo conocido (105 masculinos y 41 femeninos) con un rango de edad entre 19 y 82 años. Cuantificaron diez variables histomorfométricas de las cuales cuatro mostraron una correlación moderada con la edad: $\mathrm{N}^{\circ}$ total de osteonas $(\mathrm{r}=0.53)$, porcentaje de hueso no remodelado $(\mathrm{r}=-0.53), \mathrm{N}^{\circ}$ total de canales no haversianos $(\mathrm{r}=-0.55)$ y porcentaje promedio de hueso fragmentado $(\mathrm{r}=0.55)$. Estas variables fueron utilizadas para calcular fórmulas de regresión lineal simple y múltiple. El error estándar (ES) de la estimación oscilo entre los 13.31 y 14.04 años.

En Korea, Han y colaboradores (2009) formularon una ecuación a partir de la corteza anterior de fémur en una muestra contemporánea. Analizaron 72 piezas, 44 masculinos y 28 femeninos, cuya media de edad fue de 66.6 y 70.3 años respectivamente. Las secciones fueron teñidas con la técnica de Villanueva y cuantificaron cuatro variables: densidad poblacional osteonal, tamaño promedio osteonal, diámetro promedio del canal de Havers y ancho cortical. Las observaciones fueron realizadas con un microscopio polarizado, de 100X y las variables analizadas con un procesador de imágenes. Los resultados demostraron que el promedio del tamaño osteonal presentó una alta correlación con la edad $(\mathrm{r}=0.818)$, seguido por la densidad poblacional osteonal $(\mathrm{r}=0.810)$. Por el contrario, el ancho cortical y el tamaño de los conductos de Havers presentaron correlaciones débiles ( $\mathrm{r}=0.406$ y 0.296 , respectivamente). La ecuación propuesta por los autores arrojó un valor de $\mathrm{R}^{2}=0.789$ con un error estándar de 6.65 años.

En el ámbito nacional se han realizado aportes y adecuaciones de técnicas microsestructurales sobre tejidos duros (huesos-dientes) aplicados a estudios bioantropológicos (Restelli et al. 1997; Vasallo et al. 2000; Vasallo y Restelli, 2000; Vasallo et al. 2001; Desántolo et al. 2011).

Vasallo y colaboradores (2000) elaboraron una ecuación predictiva a partir del fémur sobre una muestra de 20 individuos, entre 15 y 98 años, del Cementerio Municipal de La Plata (La Plata, Provincia de Buenos Aires). Cuantificaron una sola variable, el porcentaje de osteonas fragmentarias $\left(\mathrm{R}^{2}=0.8611\right)$.

Recientemente, con el propósito de analizar la validez y ajuste de diferentes métodos microscópicos de estimación de edad se han elaborado ecuaciones predictivas, a partir de la diáfisis femoral, en individuos adultos (Desántolo, 2013). La muestra estudiada estuvo conformada 
por 71 individuos pertenecientes a la Colección Osteológica "Prof. Dr. Rómulo Lambre" (Salceda et al., 2012) 54 femeninos y 89 masculinos, entre 22 y 91 años con una edad media de 69.86 años. Las secciones histológicas analizadas se obtuvieron a partir de la remoción de tejido cortical de la mitad anterior de la diáfisis femoral, tal y como sugieren los estudios previamente citados. Se cuantificaron cinco variables microestructurales, de las cuales cuatro demostraron asociación significativa con la edad. El número de osteonas fragmentarias presentó una alta correlación positiva con la edad $(\mathrm{r}=0.779)$, seguida por la densidad poblacional osteonal $(\mathrm{r}=0.741)$, el porcentaje de osteonas fragmentarias $(\mathrm{r}=0.646)$ y el número de osteonas completas $(\mathrm{r}=0.330)$ que, a diferencia de la anteriores presentó una correlación positiva baja. Contrariamente, el diámetro promedio de los conductos de Havers no presentó relación lineal con la edad $(\mathrm{r}=0.158)$.

Por último, cabe mencionar que paralelamente, varios autores han descripto numerosos métodos a partir de la aplicación de otras técnicas histomorfométricas, las cuales varían de una muestra a otra, según las piezas óseas seleccionadas e.g. tibia (Hauser et al., 1980; Thompson y Galvin, 1983); húmero (Iwamoto et al., 1978; Yoshino et al., 1994; Nor et al., 2006), costilla (Cho et al. 2002; Kim et al., 2007; Pavón et al., 2010; Cannet et al., 2011), clavícula (Stout y Paine, 1992; Stout et al., 1996; Lee et al., 2014; Gomes et al., 2014), el tipo de tejido óseo utilizado (cortical o trabecular) y las estructuras microscópicas observadas (Stout, 1998) suministrando diferentes ecuaciones predictivas, específicas para cada población.

\section{Conclusión y perspectivas futuras}

Como hemos visto previamente, desde la publicación de "The microscopic determination of age in human cortical bone" el método de Kerley (1965) fue testeado en muestras independientes y modificado por varios investigadores con el propósito de subsanar y ajustar algunos inconvenientes, sobre todo, aquellos vinculados con la reproducibilidad, confiabilidad y precisión metodológica (e.g. composición y tamaño de la muestra de referencia, tipo de hueso analizado, área de muestreo, variables re- levadas, observación y cuantificación microscópica, entre otros).

Finalmente, creemos que la histomorfometría ofrece estimaciones precisas y efectivas para adultos mayores de 50 años, en contraste con la mayoría de los métodos basados en la morfología macroscópica en donde el límite de las estimaciones es, precisamente, los 50 años de edad cronológica.

Asimismo, debe enfatizarse la importancia de los estudios microscópicos en aquellos casos en los cuales los restos se encuentran fragmentados o incompletos, y resulta imposible la aplicación de los métodos convencionales. Por lo tanto, el uso de técnicas microscópicas de manera complementaria, garantizaría una mayor precisión en la estimación de edad a la muerte de restos esqueletarios en poblaciones no documentadas.

Por último, si bien la utilización de las técnicas histológicas ha presentado cierta incertidumbre respecto de su exactitud y fiabilidad, concordamos con Stout y Crowder (2012) que la estandarización de los métodos, así como la mejora de los protocolos de validación harán de la histomorfometría una herramienta útil para el análisis bioantropológico y forense.

Continuando en esta línea de investigación y en el marco del proyecto denominado "Análisis de la variación histomorfológica del esqueleto durante la ontogenia. Implicancias en el estudio de la osteobiología humana" creemos que es importante profundizar en los siguientes aspectos: 1) balancear las muestras de referencia en cuanto a la distribución de edad y sexo, dada la escasa representación de individuos jóvenes y la mayor representación de individuos masculinos, a fin de explorar dimorfismo sexual en la expresión de las variables histomorfológicas. 2) testear las diferentes ecuaciones predictivas en muestras independientes, a fin de evaluar el grado de precisión y aplicabilidad de las mismas, especialmente por ser el forense uno de los campos de aplicación; 3) analizar otros factores que influyen en la dinámica de la remodelación ósea (sexo, actividad física, patologías, entre otros) y consecuentemente en la estimación de la edad.

\section{AGRADECIMIENTOS}

Nuestro agradecimiento a los Dres. Rocío 
García Mancuso y Marcos Plischuk por sus análisis y críticas del manuscrito. Al editor y dos evaluadores anónimos por sugerencias y comentarios realizados, los cuales sin duda mejoraron la calidad del texto.

\section{LITERATURA CITADA}

Ahlqvist J, Damsten O. 1969. A modification Kerley’s method for the microscopic determination of age in human bone. J Forensic Sci 14:205-212.

Bednarek J. 2008. Methods of age at death estimation based on compact bone histomorphometry. Arch Med Sad Krym. 58(4): 197-204.

Bell LS. 2012. Histotaphonomy. En: Crowder C, Stout S, editores. Bone histology: an anthropological perspective. Boca Raton: CRC Press. p. 241-254.

Brooks ST, Suchey JM. 1990. Skeletal age determination based on the os pubis: A comparison of Acsádi-Nemeskéri and Suchey-Brooks methods. J Hum Evol. 5:227238. doi:10.1007/BF02437238

Bruce RM, Burr BN, Sharkey NA. 1998. Skeletal tissue mechanics. New York: Springer-Verlag.

Buckberry JL, Chamberlain AT. 2002. Age estimation from the auricular surface of the ilium: a revised method. Am J Phys Anthropol 119(3):231-239. doi: 10.1002/ ajpa. 10130

Cannet C, Baraybar JP, Kolopp M, Mayer P, Ludes B. 2011. Histomorphometric estimation of age in paraffinembedded ribs: a feasibility study. Int J Legal Med 125:493-502. doi:10.1007/s00414-010-0444-6

Christensen AM, Crowder CC. 2009. Evidentiary standards for forensic anthropology. J Forensic Sci 54(6):12111216. doi:10.1111/j.1556-4029.2009.01176.x

Cho H, Stout SD, Madsen RW, Streeter MA. 2002. Population-specific histological age-estimating method: a model for known African-American and EuropeanAmerican skeletal remains. J Forensic Sci 47:12-18. doi:10.1520/JFS15199J

Crescimanno A, Stout S. 2012. Differentiating fragmented human and no human long bone using osteon circularity. J Forensic Sci 57(2):287-294. doi:10.1111/j.15564029.2011.01973.x

Desántolo B, García M, Andrini L, Errecalde A, Inda AM. 2011. Aplicación de diferentes metodologías macro y microscópicas para la estimación de edad a la muerte en humanos no documentados. Libro de resúmenes del $1^{\text {er }}$ Congreso Regional de UNLAR Criminalística. XII Congreso Nacional de Criminalística y Ciencias Forenses. IX Congreso Internacional de Criminalística y Ciencias Forenses. La Rioja. (Publicación Electrónica Formato Libro).

Desántolo, B. 2013. Validación metodológica para la estimación de edad en restos óseos humanos adultos: análisis histomorfométrico. Tesis Doctoral Inédita. Facultad de Ciencias Médicas. Universidad Nacional de La Plata. Disponible en :http://www.postgradofcm.edu.ar/ ProduccionCientifica/TesisDoctorales/34.pdf

Enlow DH. 1982. Handbook of facial growth. Philadelphia: WB Saunders Company.

Ericksen MF. 1991. Histological estimation of age at death using the anterior cortex of the femur. Am J Phys Anthropol 84:171-179. doi:10.1002/ajpa.1330840207

Franklin D. 2010. Forensic age estimation in human skeletal remains: current concepts and future directions. Legal
Medicine 12:1-7. doi:10.1016/j.legalmed.2009.09.001

Fazekas IG, Kosa F. 1978. Forensic fetal osteology. Budapest: Akadémiai Kiadó.

Ferembach DI, Schwidetzky I, Stloukal M. 1980. Recommendations for age and sex diagnoses of skeletons. Workshop of European Anthropologists. J Hum Evol 9:517-549. doi:10.1016/0047-2484(80)90061-5

Ferrante L, Cameriere R. 2009. Statistical methods to assess the reliability of measurements in the procedures for forensic age estimations. Int J legal Med. 123:277-283. doi:10.1007/s00414-009-0349-4

Frost HM. 1985. The "new bone": some anthropological potentials. Year Am J Phys Anthropol 28(S6):211-226. doi:10.1002/ajpa.1330280511

Frost HM. 1987. Secondary osteon population densities: an algorithm for estimating the missing osteons. Am J Phys Anthropol 30(58):239-254. doi:10.1002/ ajpa. 1330300513

Gomes R, Jácome Hernández C, Cunha E. 2014. Un abordaje histológico para la estimación de la edad en antropología forense: un estudio preliminar. Investigación Forense III. Santiago de Chile: Servicio Médico Legal. p. 7-26.

Han S, Kim S, Ahn Y, Huh G, Kwak D, Park D, Lee U, Kim Y. 2009. Microscopic age estimation from the anterior cortex of the femur in Korean adults. J Forensic Sci. 54(3):519-522. doi:10.1111/j.1556-4029.2009.01003.x

Hauser R, Barres D, Durigon M, Derobert L. 1980. Identification par l'histomorphometrie du femur et du tibia. Acta Medicinae Legalis et Socialis 30:91-97.

Hillson S. 2005. Dental anthropology. Cambridge: Cambridge University Press.

Hillier M, Bell LS. 2007. Differentiating human bone from animal bone: a review of histological methods. J Forensic Sci 52(2):249-263. doi:10.1111/j.15564029.2006.00368.x

Iwamoto S, Oonuki E, Konishi M. 1978. Study on the age-related changes of the compact bone and the age estimation: on the humerus. Acta Medica Kinki Univ 3:203-208.

Kemkes-Grottenthaler A. 2002. Aging through the ages: historical perspectives on age indicators methods. En: Hoppa RD, Vaupel JW, editores. Paleodemography: Age distributions from skeletal sample. Cambridge: Cambridge University Press. p. 48-72. doi:10.1017/ CBO9780511542428.004

Kerley ER. 1965. The microscopic determination of age in human bone. Am J Phys Anthropol 23(2):149-164. doi:10.1002/ajpa.1330230215

Kerley ER, Ubelaker DH. 1978. Revisions in the microscopic method of estimating age at death in human cortical bone. Am J Phys Anthropol 49(4):545-546. doi:10.1002/ajpa.1330490414

Keough N. 2007. Estimation of age at death from the microscopic structure of the femur. Thesis of Master of Science in Anatomy. School of Medicine. Faculty of Health Sciences. University of Pretoria.

Keough N, L'Abbé EN, Steyn M. 2009. The evaluation of age-related histomorphometric variables in cadaver sample of lower socioeconomic status: implications for estimating age at death. Forensic Sci Int 191:114-119. doi:10.1016/j.forsciint.2009.07.012

Kim YS, Kim DL, Park DK, Lee JH, Chung NE, Lee WT, Han SH. 2007. Assessment of histomorphological features of the sternal end of the fourth rib for age estimation in Koreans. J Forensic Sci 52(6):1237-1242. doi:10.1111/j.1556-4029.2007.00566.x

Krenzer U. 2006. Compendio de métodos antropológico fo- 
renses para la reconstrucción del perfil osteo-biológico. Guatemala: Centro de Análisis Forenses y Ciencias Aplicadas (CAFCA).

Kosa F. 1989. Age estimation from the fetal skeleton. En: Iscan MY, editor. Age markers in the human skeleton. Springfield: CC. Thomas, Publishres. p. 21-54.

Lee U, Jung G, Choi G, Kim Y. 2014. Anthropological age estimation with bone histomorphometry from the human clavicle. Anthropologist 17(3):929-936.

Lovejoy CO. 1985. Dental wear in the Libben population: Its functional pattern and role in the determination of adult skeletal age at death. Am J Phys Anthropol 68:4756. doi:10.1002/ajpa.1330680105

Lovejoy CO, Meindl RS, Pryzbeck TR, Mensforth RP. 1985. Chronological metamorphosis of the auricular surface of the ilium: a new method for the determination of adult skeletal age at death. Am J Phys Anthropol 68(1):15-28. doi:10.1002/ajpa.1330680103

Luna L. 2008. Estructura demográfica, estilo de vida y relaciones biológicas de cazadores recolectores en un ambiente de desierto. Sitio Chenque I (Parque Nacional Lihué Calel, prov. de La Pampa, Argentina). BAR International Series 1886

Lynnerup N; Frohlich B, Thomsen J. 2006. Assessment of age at death by microscopy: Unbiased quantification of secondary osteons in femoral cross section. Forensic Sci Int 159:100-103. doi:10.1016/j.forsciint.2006.02.023

Madeline LA, Elster AD. 1995. Suture closure in the human chondrocranium: CT assessment. Radiology 196(3):747-756. doi:10.1148/radiology.196.3.7644639

Maat GJ, Maes A, Aarents M, Nagelkerke JD. 2006. Histological age prediction from the femur in a contemporary Dutch sample. The decrease of nonremodeled bone in the anterior cortex. J Forensic Sci 51(2):230-237. doi:10.1111/j.1556-4029.2006.00062.x

Martin RB, Burr DB, Sharkey NA. 1998. Skeletal tissue mechanics. New York: Springer-Verlag. doi:10.1007/9781-4757-2968-9

Martiniaková M, Grosskopf B, Omelka R, Vondráková M, Bauerova M. 2006. Differences among species in compact bone tissue microestructure of mammalian skeleton: use of a discriminant function analysis for species identification. J Forensic Sci 51(6):1235-1239. doi:10.1111/j.1556-4029.2006.00260.x

Meindl, RS, Lovejoy CO. 1985. Ectocranial suture closure: a revised method for the determination of skeletal age at death based on the lateral-anterior sutures. Am J Phys Anthropol 68:57-66. doi:10.1002/ajpa.1330680106

Meindl RS, Lovejoy CO. 1989. Age changes in the pelvis: Implications for palaeodemography. En: MY Isçan, editor. Age markers in the human skeleton. Springfield: CC Thomas Publishers. p 137-168.

Mulhern DM, Ubelaker DH. 2012. Differentiating human from non human bone microstructure. En: Crowder C, Stout S, editores. Bone Histology: An anthropological perspective. Boca Raton: CRC Press. p. 109-134.

Nor FM, Pastor RF, Schutkowski H. 2006. Population specific equation for estimation of age: a model for known Malaysian population skeletal remains. Mal J For Path Sci 1(1):15-28. doi:10.1177/0025802413506573

Nor FM, Pastor RF, Schutkowski H. 2014. Age at death estimation from bone histology in Malaysian males. Med Sci Law 54(4):203-208. doi:10.1520/JFS2003348

Osborne DL, Simmons TL, Nawrocki SP. 2004. Reconsidering the auricular surface as an indicator of age at death. J Forensic Sci 49:905-911. doi:10.1520/JFS2003348

Parfitt AM. 1979. Quantum concept of bone remodeling and turnover: implications for the pathogenesis of osteoporosis. Calcif Tissue Int 28:1-5. doi:10.1007/ BF02441211

Pavón MV, Cucina A, Tiesler V. 2010. New formulas to estimate age at death in Maya populations using histomorphological changes in the fourth human rib. J Forensic Sci 55(2):473-477. doi:10.1111/j.15564029.2009.01265.x

Restelli MA, Batista SL, Vasallo ML, Maliandi NE, Méndez MG, Salceda SA. 1997. Aportes de las técnicas micro y ultraestructurales sobre restos esqueletarios a la bioantropología. Actas II Jornadas Chivilcoyanas en Ciencias Sociales y Naturales de Chivilcoy. p. 123-128.

Robling AG, Stout SD. 2008. Histomorphometry of human cortical bone: applications to age estimation. En: Katzenberg MA, Saunders SR, editores. Biological anthropology of the human Skeleton. New York: Willey Liss. p. 149-182.

Salceda S, Desántolo B, García Mancuso R, Plischuk M, Inda A. 2012. The Prof. Dr. Romulo Lambre collection: an Argentinian sample of modern skeletons. HOMO 63(4):275-281. doi:10.1016/j.jchb.2012.04.002

Santo M, Lecumberry G. 2005. El proceso de medición: análisis y comunicación de datos experimentales. Río Cuarto: Universidad Nacional de Río Cuarto.

Schaefer M, Black S, Scheuer L. 2009. Juvenile osteology. A laboratory and field manual. Nueva York: Academic Press.

Scheuer L. 2002. Application of osteology to forensic medicine. Clinical Anatomy 15:297-312. doi:10.1002/ ca. 10028

Scheuer L, Black S. 2000. Developmental juvenile osteology. New York: Academic Press.

Schmeling A, Geserick G, Reinsinger W, Olze A. 2007. Age estimation. F Science Int 165(2-3):178-181. doi:10.1016/j.forsciint.2006.05.016

Schmitt A, Murail P, Cunha E, Rongé E. 2002. Variability of the pattern of aging on the human skeleton: evidence from bone indicators and implications age at death estimation. J Forensic Sci 47(6):1203-1209. doi:10.1520/ JFS15551J

Schulz R, Muhler M, Mutze S, Schmidt S, Reisinger W, Schmeling A. 2005. Studies on the time frame for ossification of the medial epiphysis of the clavicle as revealed by CT scans. Int J Legal Med 119(3):142-145. doi:10.1007/s00414-005-0529-9

Singh IJ, Gunberg DL. 1970. Estimation of age at death in the human males from quantitative histology of bone fragment. Am J Phys Anthropol 33(3):373-392. doi:10.1002/ajpa.1330330311

Skedros JG.2012. Interpreting load history in limb-bone diaphyses: important considerations and their biomechanical foundation. En: Crowder C, Stout S, editores. Bone histology: an anthropological perspective. Boca Raton: CRC Press. p. 153-220.

Stout SD. 1988. The use histomorphology to estimate age. J Forensic Sci. 33(1):121-125. doi:10.1520/JFS12442J

Stout SD. 1989. Histomorphometric analysis of human skeletal remains. En: Ișcan MY, Kennedy KR, editores. Reconstruction of life from the Skeleton. New York: Alan R. Liss, Inc. p. 41-52.

Stout SD, Crowder C. 2012. Bone remodeling, histomorphology and histomorphometry. En: Crowder C, Stout $\mathrm{S}$, editores. Bone histology: an anthropological perspective. Boca Raton: CRC Press. p. 1-22.

Stout SD, Paine RR. 1992. Brief communication: histological age estimation using rib and clavicle. Am J Phys Anthropol 87(1):111-115. doi:10.1002/ajpa.1330870110

Stout SD, Porro MA, Perotti B. 1996. Brief communication: 
a test and correction of the clavicle method of Stout and Paine for histological age estimation of skeletal remains. Am J Phys Anthropol 100(1):139-142. doi:10.1002/ (SICI) $1096-8644$ (199605) $100: 1<139:$ :AIDAJPA12>3.0.CO;2-1

Streeter M. 2012. Histological age at death estimation. En: Crowder C, Stout S, editores. Bone histology: an anthropological perspective. Boca Raton: CRC Press. p. 135-152.

Thompson DD. 1979. The core technique in the determination of age at death of skeletons. J Forensic Sci 24:902915. doi:10.1520/JFS10922J

Thompson DD, Galvin CA. 1983. Estimation of age at death by tibial osteon remodeling in an autopsy series. Forensic Sci Int 22:203-211. doi:10.1016/0379 0738(83)90015-4

Ubelaker DH. 1986. Estimation of age at death from histology of human bone. En: Zimmerman MR, Angel JL, editores. Dating and age determination of biological materials. London: Croom Helm. p. 240-247.

Ubelaker DH. 2000. Methodological considerations in the forensic applications of human skeletal biology. En: Katzenberg MA, Saunders SR, editores. Biological anthropology of human skeletal. New York: Wiley-Liss, Inc. p. 41-67.

Ubelaker DH. 2005. Estimating age at death. En: Rich J, Dean DE, Power RH, editores. Forensic medicine of the lower extremity: human identification and trauma analysis of the thigh leg and foot. Totowa NJ: Human Press Inc. p. 99-112. doi:10.1385/1-59259-897-8:099

Vasallo ML, Restelli MA. 2000. Técnica por desgaste de tejidos duros para estudios histomorfométricos. Libro de Resúmenes. IV Congreso de la Sociedad Morfológica de La Plata. p. 33

Vasallo ML; Restelli MA, Salceda SA, Méndez MG, Paggi R, Maliandi N, Batista S, Bruno M. 2000. Nueva variable para la determinación de la edad a la muerte por histomorfometría. Libro de Resúmenes del IV Congreso de la Sociedad de Ciencias Morfológicas de La Plata. p. 12.

Vasallo ML, Flores OB, Pan MF. 2001. Estimación de edad en huesos largos humanos mediante análisis escópico e histomorfométrico. Cs Morfol 5(8).

White TD, Black MT, Folkens PA. 2012. The human osteology. Elsevier Academic Press.

Watanabe Y, Konishi M, Shimada M, Ohara H, Iwamoto S. 1998. Estimation of age from the femur of Japanese cadavers. Forensic Sci Int. 98:55-65. doi:10.1016/S03790738(98)00136-4

Yoder C, Ubelaker DH, Powell JF. 2001. Examination of variation in sternal rib end morphology relevant to age assessment. J Forensic Sci 46:223-227. doi:10.1520/ JFS14953J

Yoshino M, Kazuhiko I, Sachio M, Sueshige S. 1994. Histological estimation of age at death using microradiographs of humeral compact bone. Forensic Sci Int 64(23):191-198. doi:10.1016/0379-0738(94)90231-3 